\title{
Graphene booms in factories but lacks a killer app
}

Although the wonder material is being made in record volume, commercial success is elusive.

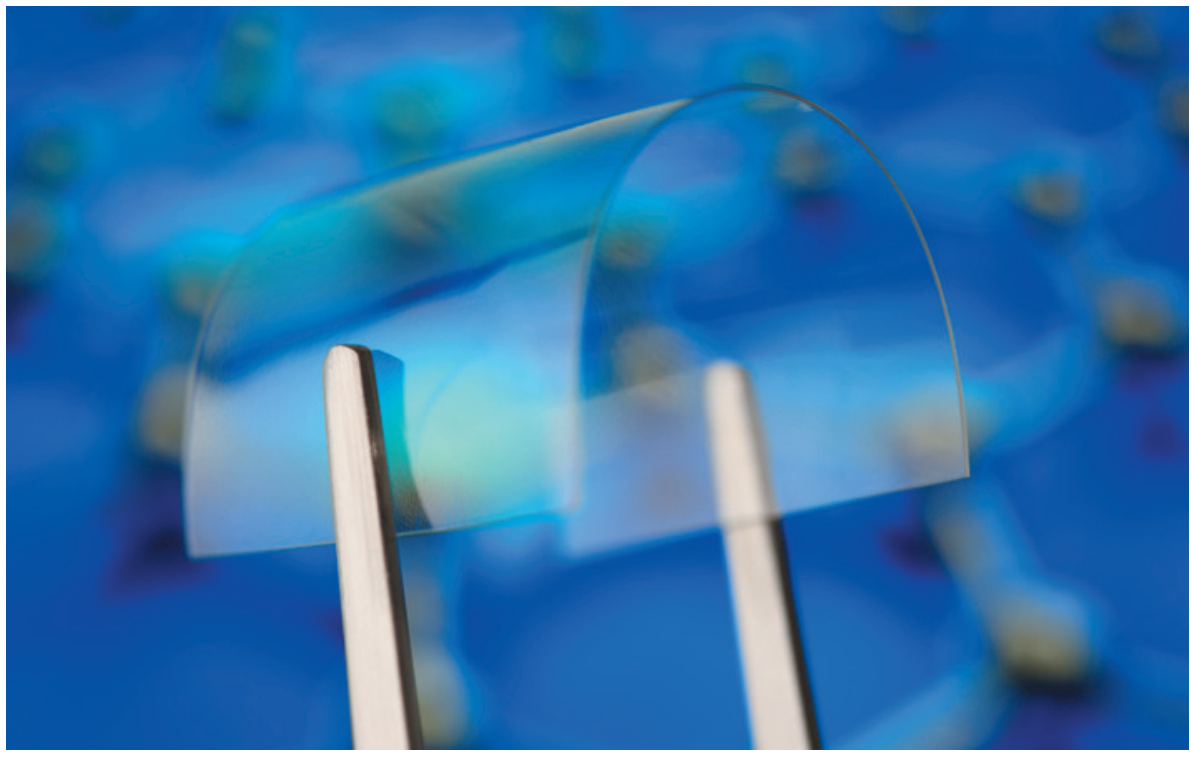

Graphene films can be used as transparent electrodes for touchscreens.

\section{BY MARK PEPLOW}

$\mathrm{T}$ he city of Manchester, UK, is gearing up for a graphene jamboree. Graphene Week 2015, which kicks off on 22 June, is sure to delight its more than 600 attendees with a conference and celebrations of the 'wonder material'. Graphene's commercial future, however, is much less certain.

The atom-thin flakes of carbon are being produced in record volume and have found their way into a handful of eye-catching gizmos. But experts fret that graphene production far exceeds requirements, and that the material offers only marginal benefits over incumbent technologies in many of its target applications.

"There's a heck of a lot of production capacity and not much demand, because we just haven't seen any compelling technologies coming through," says Ross Kozarsky, a senior analyst at market-intelligence company Lux Research, who is based in San Francisco, California.

This mirrors the trajectory of carbon nanotubes, which were once touted as transformative but have so far failed to make a significant commercial impact. "Graphene looks much closer to the next carbon nanotube than the next silicon," says Kozarsky. In a 2014 report, Lux predicted that the global market for graphene would be worth US $\$ 349$ million by 2025 ; by comparison, the University of Manchester estimates that graphene has already attracted \$2.4 billion for research (see 'Market growth').

Some scientists are growing frustrated that companies are not lining up outside their offices begging to use the material, says Helena
Theander, innovation deputy at the European Union's €1-billion (US\$1.1-billion) Graphene Flagship research programme, which was set up in 2013 to usher graphene from the lab to the marketplace within a decade.

Researchers have been in thrall to graphene's properties ever since the material was first isolated by the University of Manchester's Andre Geim and Konstantin Novoselov (K. S. Novoselov et al. Science 306, 666-669; 2004) - who won the 2010 Nobel Prize in Physics for their work. Graphene is transparent and flexible, yet very strong; electrons zoom through it; and it conducts heat ten times better than copper. Enthusiasts expect these qualities to propel graphene into dozens of commercial applications, from flexible electronics to strong, lightweight composites.

\section{GRAPHENE GLUT}

This has sparked a graphene-making frenzy, particularly in China (see 'Manufacturing fever'), where the government has poured investment into some half a dozen 'graphene industry parks', which host research institutes and business incubators side by side. Manufacturers enjoy reduced infrastructure costs, and are not expected to repay government grants. The graphene mass-produced in China falls broadly into two categories: thin films that can be used as transparent electrodes for touchscreens, for example, replacing the brittle indium tin oxide (ITO) used today; and nanoplatelets, stacks of graphene flakes that form a black powder targeted at applications such as battery electrodes, in which their high surface area may help to store more charge.

Products are starting to appear. Last year, AWIT in Wuxi, China, brought 2,000 graphene-touchscreen phones to market, and in March, Galaxy Microsystems of Shenzhen,

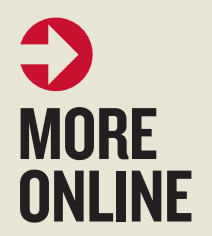

\section{TOP STORY}

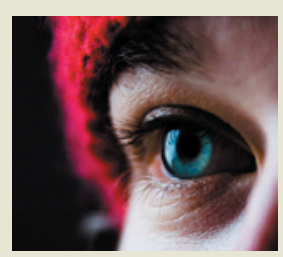

Can the human eye detect a single photon? go.nature.com/ syqcae

\section{MORE NEWS}

- The image detective who roots out manuscript flaws go.nature.com/n8ibch - Vaccine hope for post-traumatic stress go.nature.com/rxjjpe

- How gravity prevents quantum superposition on large scales go.nature. com $/ 5$ cqyhz

\section{NATURE PODCAST}

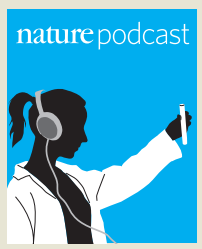

Positive memories help depression, plant intelligence, and measuring the mass of exoplanets nature.com/nature/ podcast 
China, released 30,000 phones that use graphene in their touchscreens, cases and batteries (see 'Batteries and phones rule'). But Kozarsky says that some nanoplatelet producers are selling their product at below cost to offload their surplus. And although graphenefilm factories have quoted impressive-sounding figures on capacity, these are sometimes used as a "marketing tool", says Guocai Dong, vicedean of the JiangNan Graphene Research Institute, which is in the Changzhou graphene park. "Most of the capacity is not used," he says.

One reason for the mismatch is that graphene films still do not beat ITO on price. The films are grown on hot copper foil from a feedstock of methane gas mixed with hydrogen, in a process called chemical vapour deposition (CVD). Dong says that heating the foil, maintaining clean rooms and separating graphene from the foil tend to push the final cost above 400 yuan (US\$64) per square metre, about twice what it needs to be to compete with ITO. And although graphene's flexibility could add value to wearable devices, the wonder material will have to beat off other pretenders to ITO's throne, including silver-nanowire meshes, which have been in development for longer.

A new approach to manufacturing is needed to depress costs further, says Xuesong Li, who helped to develop the copper-foil CVD method (X. Li et al. Science, 324, 1312-1314; 2009). Last year, Li founded LasLumin, a company in New York City that aims to slash graphene manufacturing costs by using feedstocks that are easier to handle than the potentially explosive mixture of methane and hydrogen. Graphene films may find more-profitable applications in innovative chemical sensors, rather than in taking on an established material such as ITO, he adds.

\section{FROM LAB TO MARKET}

China also dominates the graphene patent landscape (see 'Patent kings'). SIO Grafen, an innovation programme in Gothenburg, Sweden, that supports industrial graphene development, reported in March that Chinese entities hold $45 \%$ of all graphene patents granted in the seven categories that it surveyed. That is more than twice the share of China's nearest rival, the United States. However, most of the Chinese patents are registered nationally rather than globally, and they tend to be cited less often than the US ones, suggesting that they may not have much impact on graphene's long-term commercial development.

Enter the Graphene Flagship, which plans to focus on quality and innovation. With that in mind, Theander has run seven Graphene Connect workshops to introduce small and medium-sized companies to graphene researchers, and will host another during Graphene Week. But she says it is too soon for concrete results: "Growing trust and new relationships takes time, so I imagine it will take a couple of years until we have true success stories."

A similar approach has proved successful

\section{THE GRAPHENE COUNT}

\section{Manufacturing fever}

Graphene production capacity has exploded in Asia, driven mainly by China.

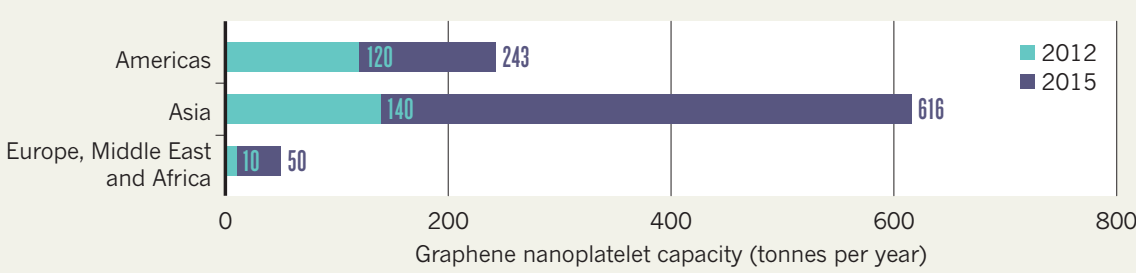

\section{Patent kings}

China dominates the graphene patent landscape in key fields, but its patents are overwhelmingly domestic and may have little global impact.

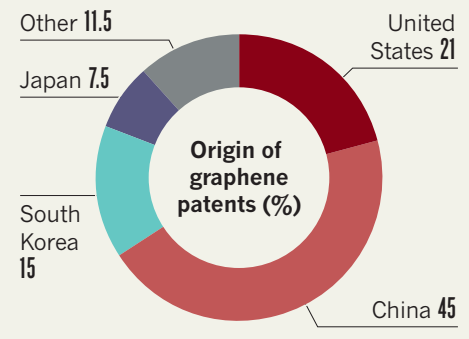

Batteries and phones rule

The most patents filed for graphene applications concern energy storage and composite materials.

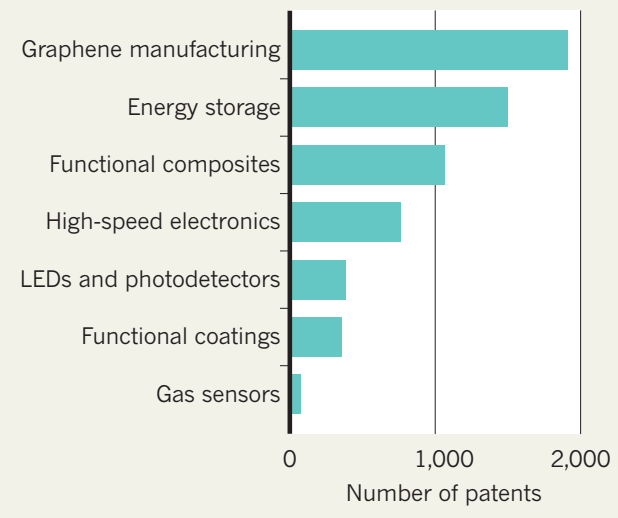

at the University of Manchester's National Graphene Institute (NGI), says its business director, James Baker. The NGI has almost 40 industrial partners who have funded work at the university; the biggest, BGT Materials in Manchester, has a subsidiary called Graphene Lighting that unveiled a 'graphene light bulb' in March. The bulb is actually a conventional light-emitting diode (LED) coated with a transparent film of graphene that draws off heat more effectively than conventional metal components, improving the efficiency and longevity of the LED. The company hopes that the bulb will go on sale in the next few months.

Meanwhile, Baker notes, multinational sports-equipment company Head has unveiled a range of tennis rackets that are strengthened by a dash of graphene in their frames.

Such niche applications are a start, but if graphene is to fulfil the commercial potential anticipated by projects such as the Graphene
Flagship, it will need to find a role in which it dramatically and reliably outperforms existing technologies at a reasonable cost.

Baker believes that the answer lies in creating devices that rely on a combination of graphene's properties - flexible electronics, for example - or that could have a transformative global impact, such as in membranes that desalinate water. But it is early days, he says: "There are so many potential applications that it's difficult to see what will be the killer app." - SEE NEWS FEATURE P.274 AND BOOKS \& ARTS P.284

\section{CORRECTION}

The News story 'Start-ups fight for a place in Boston's biotech hub' (Nature 522, 138139; 2015) misstated prices for lab and office space in Kendall Square; the figures reflect annual rather than monthly rents. 